\title{
A submm survey of high-redshift galaxy clusters: a submm Butcher-Oemler effect?
}

\author{
Tracy Webb ${ }^{1}$, H. K. C. Yee ${ }^{2}$, Henk Hoekstra ${ }^{2,3}$ \\ and Mike Gladders ${ }^{4}$ \\ ${ }^{1}$ Leiden Observatory, Niels Bohrweg 2, 2333CA Leiden, The Netherlands email: \\ webb@strw.leidenuniv.nl \\ ${ }^{2}$ Department of Astronomy \& Astrophysics, University of Toronto, 60 St. George St., Toronto, \\ M5S 3H8, Canada \\ ${ }^{3}$ Canadian Institute for Theoretical Astrophysics, University of Toronto, 60 St. George St., \\ Toronto, M5S 3H8, Canada \\ ${ }^{4}$ Carnegie Observatories, 813 Santa Barbara St., Pasadena, CA, 91101, USA
}

\begin{abstract}
We present the initial results of a deep submillimeter survey of ten $z \sim 1$ galaxy clusters with the SCUBA bolometer array on the JCMT. Recently, an excess number of dust enshrouded starbursts in high-redshift galaxy cluster fields was reported (Best 2002) and has been interpreted as increased star-formation within young clusters. An alternative explanation is that these starbursts are background objects behind clusters with enhanced lensing crosssections, over that predicted from simple lensing models, so-called "super-lenses" seen in recent optical cluster surveys. To investigate this issue, we are studying a set of clusters which show strong optical arcs (super-lenses) and a set of clusters of equal redshift and richness but which are not super-lenses. The preliminary results, based on $50 \%$ of the sample, tentatively suggest that, while the dusty starbursts are limited to the super-lensing cluster fields, they are not background objects but lie within the clusters themselves. Cluster-cluster merging offers a means of increasing both the lensing cross-section, thereby accounting for the multiple strong optical arcs, and increasing the star formation within the clusters through galaxy interactions.
\end{abstract}

\section{Introduction}

Submillimeter (submm) observations of the high redshift universe provide a unique and crucial window on the formation and evolution of galaxies (Eales et al. 2000, Webb et al. 2003). They trace regions of extreme luminosity and star-formation and, in particular, are sensitive to dusty systems which may be completely obscured at optical wavelengths. Furthermore, the unique negative $K$-correction produces an approximately constant observed $850 \mu \mathrm{m}$ flux with redshift; therefore submm-luminous systems above a given intrinsic luminosity can be detected to very high redshifts of $z \sim 6$. Thus, submm observations offer a potentially powerful tool with which to study the formation and evolution of galaxies, and the formation of the massive ellipticals in clusters, which likely formed in intense and dusty starbursts at high redshifts.

Submm astronomy is still in its infancy and only a handful of submm facilities exist which are capable of carrying out useful submm observations. The program presented here has used the Submm Common User Bolometer Array (SCUBA) on the James Clerk Maxwell Telescope (JCMT) in Hawaii, operating at $850 \mu \mathrm{m}$. Above a redshift of $z \sim 1$ the galaxies detected with SCUBA have far-infrared (FIR) luminosities in excess of $10^{12} L_{\odot}$, and are similar to the ultraluminous infrared galaxies (ULIRGs) in the local universe. Like present day ULIRGs, they harbour a powerful energy source of either intense star formation and/or an active galactic nucleus, which is so deeply enshrouded by dust that 
the bulk of their energy is absorbed by the dust and re-emitted at FIR wavelengths. The flux limit of SCUBA is set by the confusion limit of the telescope at $850 \mu \mathrm{m}, S_{850 \mu m} \sim$ $2 \mathrm{mJy}$, below which individual sources cannot be resolved. However, a number of groups have employed galaxy clusters as gravitational lenses to stretch the source plane and push the confusion limit to deeper levels (Smail et al. 2002). At low redshift $(z<0.5)$ where clusters are dominated by old, early-type galaxies with little or no dust and star formation, clusters do not contribute to the submm source counts and this is a relatively clean method of sampling the background population.

The recent observations of Best (2002) however show a possible excess of submm sources toward $z \sim 1$ cluster fields over that expected from simple isothermal sphere lensing models. Since lensing appears insufficient to explain the counts, this has been interpreted as an increase in dusty star formation in high redshift clusters, that is, a submm Butcher-Oemler effect. An increase in star formation in higher redshift galaxy clusters is not a new idea (Butcher \& Oemler 1984) but the star formation rates implied by the $850 \mu \mathrm{m}$ luminosities are extreme $\left(>100 \mathrm{M}_{\odot} \mathrm{yr}^{-1}\right)$ and must be caused by previously unstudied processes. In general the processes which act to enhance or stifle star formation in cluster and infalling galaxies are poorly understood and the existence of such intense star bursts add a new dimension to the problem. However, the number of clusters studied by Best (2002) is very small, and the increase in dusty starburst in high-redshift clusters is by no means an established global effect but must be verified through larger samples.

The above cluster-member interpretation rests on the fact that there are more submmluminous systems than expected from simple lensing considerations, but if the lensing is stronger than the simple models predict, these systems could be highly magnified background objects. In fact, there is mounting evidence that some clusters at $z \sim 1$ do have enhanced lensing cross-sections. In the 90 square degree Red-sequence Cluster Survey (RCS, Gladders \& Yee 2000), Gladders et al. (2003) found a fraction of multiple optical arcs for clusters at $z>0.6$ much larger than expected from a $\Lambda$ CDM cosmology. This, along with similar results from other high/medium redshift cluster samples (e.g. Bartlemann et al. 1998), leads to the interpretation that at higher redshift there is a subset of rich clusters which have much higher lensing cross-sections than that estimated from a standard isothermal mass model. These "super-lenses" could be due to substructure, orientation effects, or a high central mass surface density, and could account for the excess submm sources seen toward some clusters.

\section{The RCS submm survey: observations and preliminary results}

We have begun an observational program with SCUBA at $850 \mu \mathrm{m}$ to verify the results of Best (2002) and, if a true excess exists, to determine if the objects are primarily background lensed systems or objects at the same redshift as the cluster, either proper cluster members or infalling galaxies. We are imaging a sample of ten $0.6<z<1.1$ clusters selected from the RCS, which has produced a large and systematically selected sample of $z \sim 1$ clusters (see also Blindert et al. and Yee et al., these proceedings). In order to address the question of whether this effect is limited to strong lensing clusters we have selected 5 strong-lensing clusters (i.e., those that show strong optical arcs) and 5 clusters of equal richness and redshift but which do not show enhanced strong lensing.

We reach a $3 \sigma$ depth of $\sim 3$ mJy which requires $\sim 10$ hours of integration per cluster. The field of view of SCUBA is $\sim 2.5 \times 2.5 \mathrm{arcmin}^{2}$ and is well matched to the inner regions of the cluster fields (that is, within the virial radius). This is the approximate flux density that Arp 220 (a typical ULIRG in the local universe) would have if placed at the cluster redshifts and thus we are sensitive to extreme dusty star bursts. At such a depth we 
have essentially reached the confusion limit at $850 \mu \mathrm{m}$ and therefore little is gained by integrating deeper. The maps are reduced using the standard SURF pipeline and the noise is estimated and sources extracted following the technique of Eales et al. (2000). We have completed five clusters to date and the maps are shown in Figure 1 with signalto-noise contours highlighting the significant objects. There are three clusters with strong optical arcs (super-lenses), RCS0224, RCS2319, and RCS1419, and two non-super-lenses, RCS1122 and RCS1326.

\section{Discussion: cluster-cluster mergers?}

All five clusters show a single detection near the center of the cluster (roughly $(0,0)$ in Figure 1). These central sources could be detections of the central galaxies (Edge et al. 1999) or background lensed galaxies. The lensing is strongest within the inner $\sim 20$ arcsec and it is also within this region that the optical arcs are found. Unfortunately, the relatively large beam size of the JCMT at $850 \mu \mathrm{m}$ (15 arcsec FWHM) introduces an uncertainty in the submm position of up to 8 arcseconds. This, and the fact that the counterparts are dusty and likely optically faint means that it is not possible to unambiguously identify the source of the submm emission using submm and shallow optical data alone, but requires deep optical, near-infrared and radio imaging.

For the two non-super lenses, RCS1122 and RCS1326, the single central submm source is the only one detected with significance (the objects visible at the very edges of these images are too close to the edge to be considered secure detections). However, the three super-lensing clusters contain additional objects further out from the center which are significantly brighter than the central object ( $4 \mathrm{mJy}-9 \mathrm{mJy})$. All of these systems are 1.0-1.5 arcminutes from the center of the cluster where, given simple lensing considerations, the magnification is expected to be very small $(<1.2)$ and it therefore seems unlikely that these systems are lensed unless significant substructure exists, and there is currently no evidence for this in the present data.

A more likely scenario, based on the current limited evidence, is that these systems are cluster members which are undergoing very intense starbursts due to the effects of the cluster environment and interactions with other galaxies. However, the fact that the submm luminous systems are only seen in the super-lenses implies that something is still unique about these particular clusters, and suggests a common cause for the enhanced lensing cross-section (as indicated by the multiple optical lensed arcs) and the excess submm sources in the outskirts. We note that we use the term outskirts loosely as the submm sources, though far from the central region of strong lensing, are still within the virial radii of the clusters, though we have no information regarding the extent of their distribution beyond the limits of the SCUBA maps.

Cluster-cluster merging offers a possible explanation for both of these phenomena. In the standard paradigm of structure formation clusters of galaxies form hierarchically through major mergers and the accretion of smaller clusters and groups. Clusters in the local universe are, for the most part, relaxed and virialized, but at higher redshift, where clusters are younger and still forming, these processes will become important. Cluster merging and accretion must also have a profound effect on the galaxies within clusters and may be one of the dominant processes at high redshift which drives their evolution.

Torri et al. (2004) have developed a model which shows that during a major cluster merger the lensing cross section is significantly, though temporarily, increased. This lensing enhancement can bring the number of multiple optical arcs seen in the RCS and other cluster surveys into agreement with the predictions of $\Lambda$ CDM models. The effect on the cluster galaxies is not well understood though a cluster-cluster merger likely leads to 


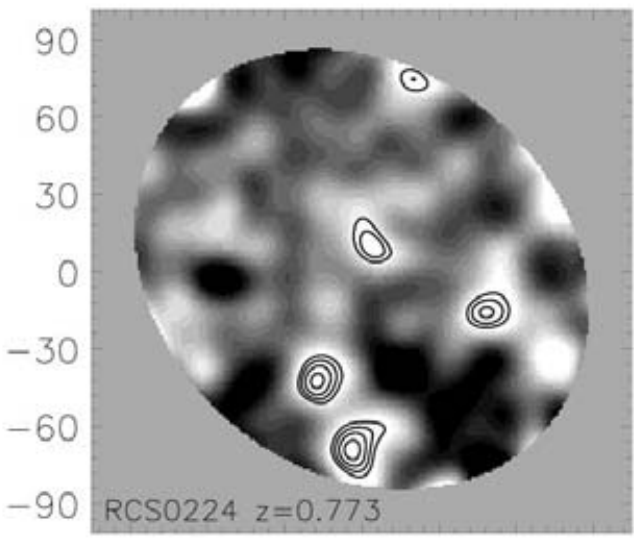

$90 \quad 60 \quad 30 \quad 0-30-60-90$

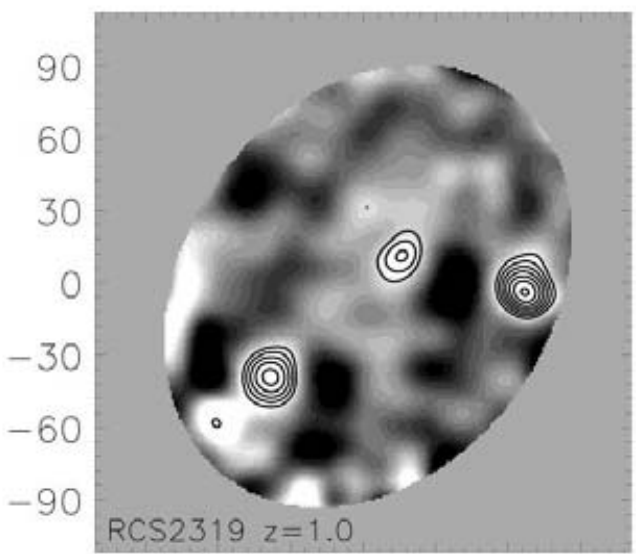

$90 \quad 60 \quad 30 \quad 0-30-60-90$
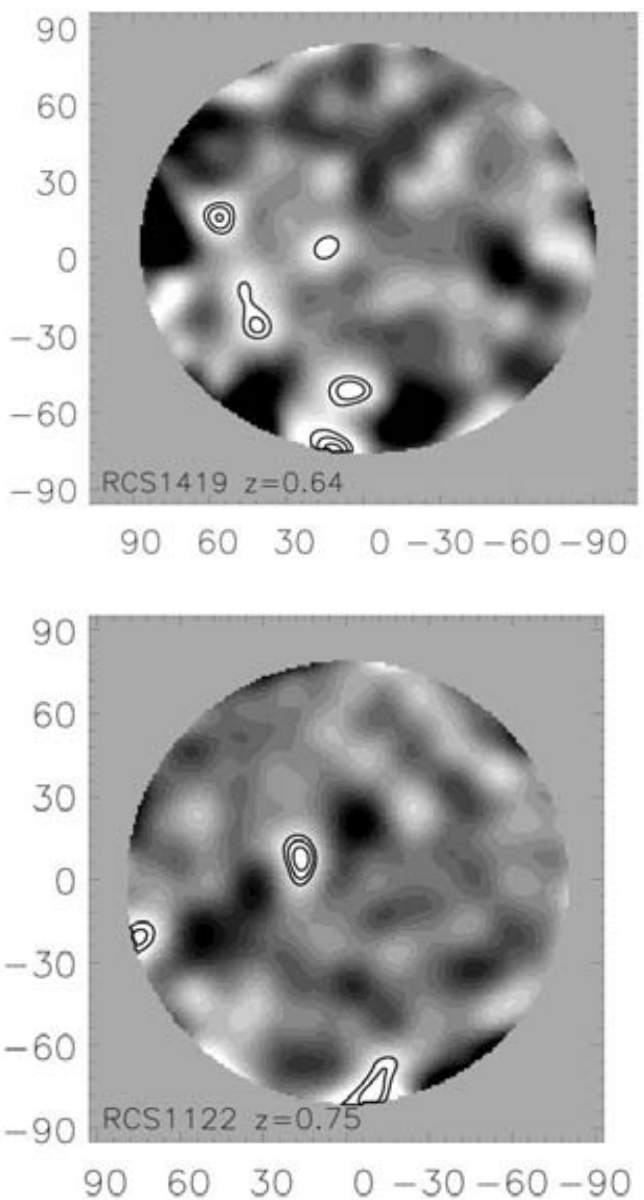

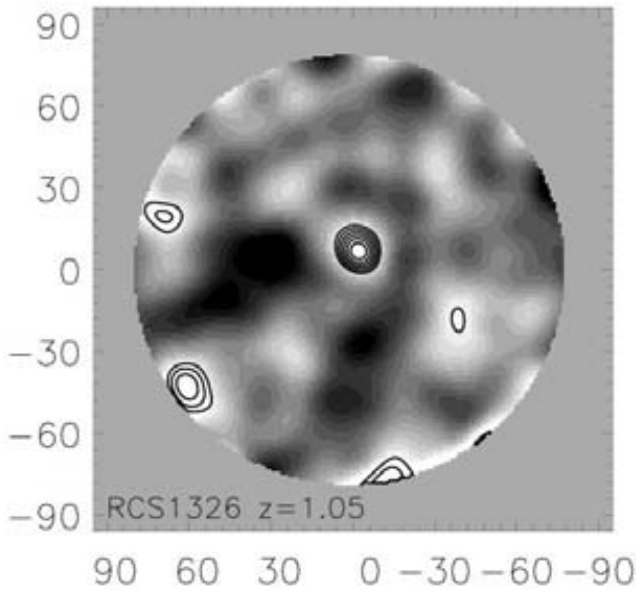

Figure 1. The $850 \mu \mathrm{m}$ maps of five RCS clusters with complete SCUBA imaging. The grey scale images correspond to the $850 \mu \mathrm{m}$ flux, and overlaid are signal-to-noise beginning at $2.5 \sigma$ and increasing in $0.5 \sigma$ steps for all clusters expect for RCS2319, which has $1 \sigma$ contour steps. The images have been smoothed with a 14 arcsec Gaussian. The strong lensing clusters are RCS0224, RCS2319, and RCS1419. Note the large number of detections in these three clusters relative to the two non-strong-lensing clusters. This will be verified with larger number statistics upon completion of the survey. 
an increased interaction rate between galaxies (see Mihos 2004 for discussion). Though major galaxy mergers are negligibly rare within cluster environments due to the high velocities of the interactions, this may not be the case in the outskirts of clusters during a merging event, where the galaxy-galaxy merger rate may increase and possibly lead to ULIRG-like star-bursts as seen in the field. Indeed, extreme starbursts have been seen in the mid-infrared studies of $z \sim 0.5$ clusters with ISO (e.g., Duc et al. these proceedings), though the trigger of these bursts is not clear.

If this scenario is correct we are observing the ongoing formation of clusters and in particular have caught some of the cluster galaxies in the process of forming the bulk of their stars. As we push cluster observations to higher redshift this effect will become increasingly important as the clusters will be younger, merging and accretion more common, and the galaxies themselves younger and possibly more gas rich. While imaging a large sample of galaxies over a broad redshift range is currently limited by the mapping speed of SCUBA (and the dearth of clusters at $z>1$ ), the commissioning of the SCUBA2 array on the JCMT in 2006 will allow wide field and deep submm observations of large samples of high-redshift clusters in very reasonable amounts of time.

\section{Ongoing and future work}

We have completed the submm imaging for $50 \%$ of the cluster sample, or five clusters, and have proposed to the JCMT to complete the remaining five clusters by January 2005. With a sample of 10 clusters we will be able to determine statistically if an excess of submm galaxies is found in high-redshift cluster fields and in strong lensing $z \sim 1$ clusters in particular. The success of this program however depends crucially on extensive followup data. As previously mentioned, the large beam of the JCMT at $850 \mu \mathrm{m}$ produces an uncertainty in the submm position and deep optical, near-infrared and radio imaging are required to identify the counterparts. Without this information we cannot study the nature and cause of the starburst or place the object behind or within the cluster through a secure redshift. Deep multi-wavelength imaging is also necessary to investigate our suggestion that the super-lensing clusters are undergoing major cluster mergers, while the non-super-lenses are not. To this end we have begun follow-up imaging on a number of clusters with the VLA, CFHT, HST, VLT and Chandra, and will continue to pursue further observations in the future for the entire cluster sample.

\section{References}

Best, P. 2002 MNRAS 336, 1293.

Bartelmann, M., Andreas, H., Colberg, J.M., Jenkins, A., \& Pearce, F.R. 1998 A\&SA 330, 1.

Butcher, H. \& Oemler, A. 1984, ApJ, 285, 426.

Eales, S., Lilly, S.J., Webb, T., Dunne, L., Gear, W., Clements, D., \& Yun, M. 2000, ApJ 120, 2244.

Edge, A.C., Ivison, R.J., Smail, I., Blain, A.W., \& Kneib, J.P. 1999, MNRAS 306, 599.

Gladders, M. \& Yee, H.K.C. 2000 AJ, 120, 2148.

Gladders, M.D., Hoekstra, H., Yee, H.K.C., Hall, P., \& Barrientos, L.F. 2003, ApJ 593, 48.

Mihos, C. 2004, In Clusters of Galaxies: Probes of Cosmological Structure and Galaxy Evolution, (ed. J.S. Mulchaey, A. Dressler, and A.Oemler), p. 278.

Smail, I., Ivison, R.J., Blain, A.W., \& Kneib. J.-P. 2002 MNRAS 331, 495.

Torri, E., Meneghetti, M., Bartelmann, M., Moscardini, L., Rasia, E., \& Tormen, G. 2004 MN$R A S$ submitted.

Webb, T., et al. 2003, ApJ 587, 41. 